\title{
Perimesencephalic and sulcal subarachnoid haemorrhage: an interesting presentation of posterior reversible encephalopathy syndrome
}

\author{
Rajesh Shankar lyer, Ramakrishnan Tirupur Chinnappan Ramalingam, Saleem Akhtar, \\ Karunakaran Muthukalathi
}

Department of Neurology, KG Hospital, Coimbatore, Tamil Nadu, India

\section{Correspondence to Dr Rajesh Shankar lyer, dr_rsh@hotmail.com}

Accepted 16 January 2018
Check for updates

\begin{tabular}{l}
\hline To cite: lyer RS, \\
Ramalingam RTC, Akhtar S, \\
et al. BMJ Case Rep \\
Published Online First: \\
[please include Day Month \\
Year]. doi:10.1136/bcr-2017- \\
222875 \\
\hline
\end{tabular}

\section{DESCRIPTION}

A 38-year-old man with hypertension presented with sudden-onset headache and vomiting. He was irritable and had a blood pressure of $180 / 120 \mathrm{~mm}$ $\mathrm{Hg}$. There were no meningeal signs or focal neurological deficits. His optic fundi were normal. Initial evaluation with CT of the brain showed subarachnoid haemorrhage $(\mathrm{SAH})$ in the right parasagittal sulcal region (figure $1 \mathrm{~A}$ ) and the left perimesencephalic cistern (figure 1B). A CT angiogram (CTA) followed by a digital subtraction angiogram (DSA) ruled out aneurysms (figure $1 \mathrm{C}-\mathrm{F}$ ). The venous phase of DSA (figure $1 \mathrm{G}$ ) and magnetic resonance (MR) venogram (figure $1 \mathrm{H}, \mathrm{I}$ ) were normal. MRI of the brain (figure 2) showed hyperintense lesions in the bilateral parieto-occipital regions and the basal ganglia suggesting posterior reversible encephalopathy syndrome (PRES). He was managed with antihypertensives and made a gradual and complete recovery.

Our patient presented with non-traumatic $\mathrm{SAH}$ on an emergent head CT scan. It showed

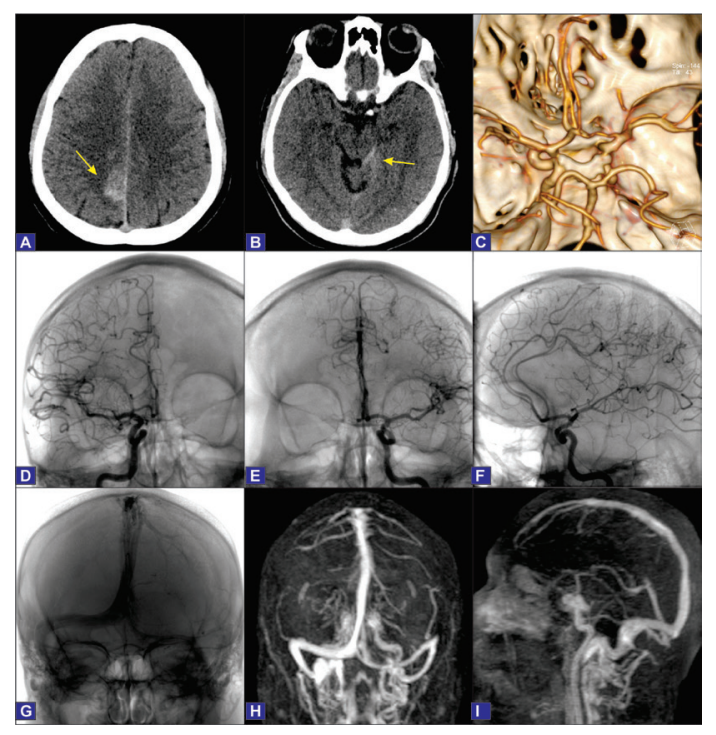

Figure $1 \mathrm{CT}$ of the brain showing right parasagittal sulcal $(A$, arrow) and left perimesencephalic subarachnoid haemorrhage $(B$, arrow). CT angiography $(C)$ was negative for aneurysms. Digital subtraction angiography (DSA) with selective right carotid injection, anteroposterior (D) and lateral (F) views, and left carotid injection (E) ruled out aneurysms. The venous phase of DSA (G) and magnetic resonance venogram $(H, I)$ were normal. simultaneous presence of blood in two interesting locations. The first is the perimesencephalic region, where the majority of cases are negative for aneurysms and is considered due to venous rupture. ${ }^{1}$ Around $10 \%$ of spontaneous SAH presents this way. Very rarely they can be due to ruptured vertebrobasilar aneurysms, cervical spinal arteriovenous malformations or dural arteriovenous fistulas.

Another distinct category of spontaneous SAH accounting for around $7 \%$ is the peripheral convexal SAH, where the bleeding is localised to a few sulci of the cerebral convexities without bleeding elsewhere. This again is a non-aneurysmal form of SAH predominantly due to cerebral amyloid angiopathy in the elderly and reversible cerebral vasoconstriction syndrome (RCVS) in the young. ${ }^{2}$ The other causes are cerebral venous sinus thrombosis, atherosclerotic large-vessel occlusion, infections, coagulation disorders, moyamoya disease, cerebral vasculitis and PRES.

Studies have reported haemorrhage in PRES in $5 \%-17 \%$ of patients. ${ }^{3}$ They present either as sulcal $\mathrm{SAH}$, minute parenchymal haemorrhages or focal haematomas in the brain parenchyma. Perimesencephalic cistern is an unreported site for bleeding due to PRES. The mechanism of haemorrhage in PRES is uncertain and controversial. Severe hypertension and the associated impaired cerebral autoregulation and rupture of pial vessels may be one mechanism. However haemorrhage is found to be equally common in normotensive patients developing PRES. The other mechanism is the postischaemic reperfusion injury following vasoconstriction of medium-sized and large-sized vessels occurring in PRES.

The possibility of vasospasm after SAH was considered. This usually develops between the fourth and ninth day after SAH, and we had completed our investigations before this time. There were also no angiographic changes compatible with vasospasm. In comparison with aneurysmal SAH, non-aneurysmal SAH especially the perimesencephalic variety has a low incidence of vasospasm.

The other issue here is the ideal imaging modality to be adopted when we deal with SAH in these two interesting locations occurring simultaneously in the same patient. A high-quality CTA is enough to identify the rare possibility of vertebrobasilar aneurysms in perimesencephalic haemorrhage. MRI of the brain with MR venography may be sufficient 


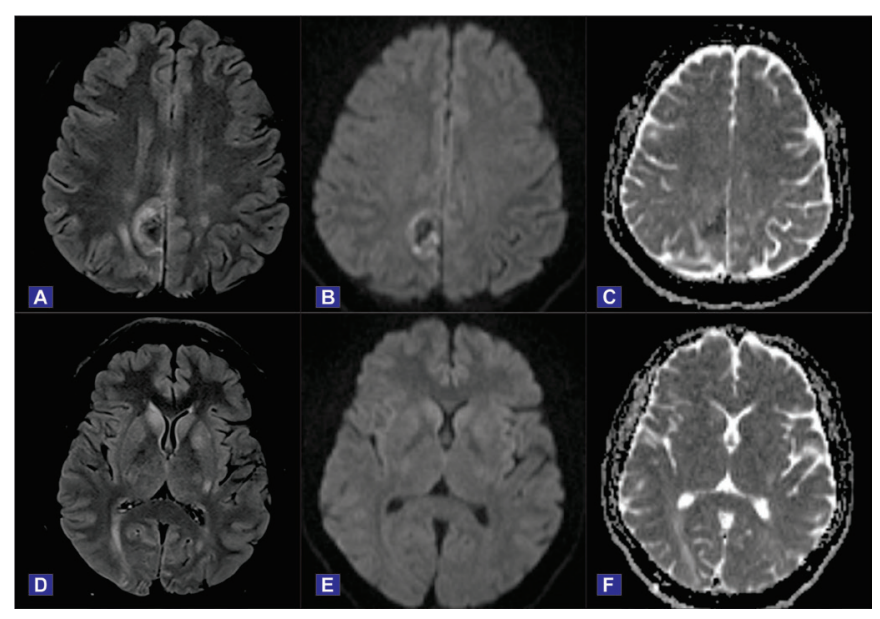

Figure 2 MRI of the brain fluid-attenuated inversion recovery (FLAIR) images ( $A, D)$ showing hyperintense lesions over the bilateral parieto-occipital and basal ganglial regions suggestive of posterior reversible encephalopathy syndrome. The diffusion-weighted images $(B, E)$ and the corresponding apparent diffusion coefficient images (C, F) show no diffusion restriction.

as the initial diagnostic work-up in convexal SAH. Vascular imaging may be necessary to detect RCVS and vasculitis. Since both the locations are known to be more commonly associated with non-aneurysmal SAH, their combined presence on either side of the brain should only strengthen this association. We probably should have opted for MRI after the CTA and could have avoided DSA. Our team wanted to rule out the possibility of a small anterior cerebral artery aneurysm causing the parasagittal bleed and went ahead with DSA. It is prudent to conclude that non-invasive imaging modalities including parenchymal and vascular imaging are sufficient in such situations, and DSA be performed only if the non-invasive tests prove inconclusive.

\section{Learning points}

- Perimesencephalic and sulcal subarachnoid haemorrhages are more commonly non-aneurysmal.

- Posterior reversible encephalopathy syndrome can present as perimesencephalic and sulcal subarachnoid haemorrhages in an emergent CT scan.

- Non-invasive imaging modalities of the brain looking for parenchymal and vascular abnormalities are generally sufficient to clinch the diagnosis in such a presentation.

Contributors RSI was involved in the design, data acquisition, interpretation and the intellectual content of the draft. RTCR and SA contributed towards intellectual content. KM contributed to the analysis and interpretation of the images.

Competing interests None declared.

Patient consent Obtained.

Provenance and peer review Not commissioned; externally peer reviewed. (c) BMJ Publishing Group Ltd (unless otherwise stated in the text of the article) 2018. All rights reserved. No commercial use is permitted unless otherwise expressly granted.

\section{REFERENCES}

1 Marder CP, Narla V, Fink JR, et al. Subarachnoid hemorrhage: beyond aneurysms. AJR Am J Roentgenol 2014;202:25-37.

2 Kumar S, Goddeau RP, Selim MH, et al. Atraumatic convexal subarachnoid hemorrhage: clinical presentation, imaging patterns, and etiologies. Neurology 2010;74:893-9.

3 Hefzy HM, Bartynski WS, Boardman JF, et al. Hemorrhage in posterior reversible encephalopathy syndrome: imaging and clinical features. AJNR Am J Neuroradiol 2009:30:1371-9.

Copyright 2017 BMJ Publishing Group. All rights reserved. For permission to reuse any of this content visit http://group.bmj.com/group/rights-licensing/permissions.

BMJ Case Report Fellows may re-use this article for personal use and teaching without any further permission.

Become a Fellow of BMJ Case Reports today and you can:

- Submit as many cases as you like

- Enjoy fast sympathetic peer review and rapid publication of accepted articles

- Access all the published articles

Re-use any of the published material for personal use and teaching without further permission

For information on Institutional Fellowships contact consortiasales@bmjgroup.com

Visit casereports.bmj.com for more articles like this and to become a Fellow 